\title{
Aircrafts Monitoring and Early Warning Based on Air Traffic Complexity Measure Analysis
}

\author{
Dong Bing ${ }^{1,2, *}$ \\ ${ }^{1}$ School of Transportation and Logistics, Southwest Jiaotong University Chengdu, Sichuan, 610031, China \\ ${ }^{2}$ Department of Graduate Student, Civil Aviation Flight University of China, Guanghan Sichuan 618307, China
}

\begin{abstract}
This paper studied air traffic complexity by the theory of complex analysis. The calculation system of air traffic complexity measure and the robustness analysis of air traffic flow were proposed. When the aircraft influenced the air traffic flow complexity mostly, the flight warning system began to work. This paper discussed the theory of the monitoring and early warning based on the complexity of air traffic complexity measure analysis. The studies shown that the method provided in this paper could discern the state parameter associated through technical indicators from complex, uncertain data in the calculation of system complexity, and the research also pointed out the flights which had larger effects on the air traffic through robustness change. The researches show that the complexity theory proposed in this paper can carry on monitoring and early warning on flights through the complexity measure and the air traffic complexity analysis.
\end{abstract}

Keywords: Air traffic complexity, Robustness, Air traffic flow, Information entropy.

\section{INTRODUCTION}

Aerospace system is a classic complex giant system, which is composed of aerospace structure, air traffic flow consisting of aircraft in operation, air traffic control rules and programs, as well as management and controller' ability. The complexity of air traffic management is up to three factors: 1.The mutual influence among people, equipment, environment and management; 2. Partial but not complete certainty of the system's condition during air traffic operation; 3 . The difficulty of measuring data to evaluate the system objectively, which needs more than qualitative analysis based on experts' research and scoring as well as flight statistics. The complexity of air traffic is a quantitative result under the interaction between aircraft-consisted air traffic flow and aerospace structure. The management of air traffic complexity is a reliable method to handle comparative intense air traffic in order to identify the complicated air traffic condition and take effective actions including operation programs to scale down the complexity to an acceptable range or level with key factors which matter to the detected complexity. Based on that, it is a significant problem that how to quantify the predicted air traffic flow complexity.

So far the most representative measures of air traffic complexity are the means of dynamic density [1] and the means of traffic intrinsic measure [2]. The conception of dynamic density (DD) is put forward by research institutions with National Aeronautics and Space Administration (NASA) as representative [3-5]. The methods of dynamic density is to indicate air traffic complexity with aircraft density, confrontation factors, air traffic controller's intention and other parameters, whose changes in value is the fundamental cause lead to the changes in controllers' workload. However, individual differences of controllers could make it hard to measure their workload accurately. The methods of traffic intrinsic measure $[6,7]$ is to describe the complexity of traffic situation evolution on the basis of traffic's intrinsic nature, such as aircraft speed and heading. Geometric disorder analysis [8], analysis of topological entropy in dynamic system [9], traffic flow disturbance analysis [10] and traffic flow proximity analysis [11] are typical ways of the traffic intrinsic measure. As the hot spot in air traffic complexity study, such kind of methods makes use of geometric measure model and dynamic system model for measure analysis. With system theory and complexity science developing in leaps and bounds, researchers represented by Bui and Amor [12] established aerospace model by the means of complex system research method, analyzed aerospace performance through evolution process qualitatively and quantitatively and proved the emergent phenomena appeared in air traffic, that is, aerospace system generates phase change phenomena when it reaches certain critical value.

It is difficult to study air traffic management system by analyzing established mathematical model because it is a non-linear, complex giant system. Therefore, the approach of non-linear time sequence analysis attracts many scholars' attention. Literature [13] proposed an air traffic complexity evaluation method based on probabilistic trajectory prediction by using stochastic linear hybrid system theory. Literature [14] raised a measure of air traffic complexity that is to estimate tracks by using the discrete state at a given time in the stochastic linear hybrid system to simulate uncertain 
aircraft positions, with restrictive conditions expressed in the manner of probability taken into account. But this method demands heavy calculation work and is apt to change by uncontrollable effects.

Entropy of information and mutual information come from information theory and are widely used in all kinds of non-linear system and complex system study [15]. The initial definition of complexity measure was raised by Kolmogorov in 1965, and then developed into concrete complexity arithmetic by Lempel and Ziv [16]. Marczyk proposed the definition that complexity measure is the information measure of structured system [17], as well as a model independent calculation method which means to conduct complexity measure used in the study of non-linear system.

Based on the summary of studies mentioned above, this article intends to solve air traffic complexity management issue and unfold the nature of air traffic dynamic process objectively, accurately and integrally by means of non-linear, unbalanced and dynamic thinking and systematic ways, with ATM workload estimation in the perspective of dynamic system complexity and non-linear time sequence information measure that is described with important parameters including Entropy of information, mutual information and complexity. It puts forward with metrization analysis system on air traffic complexity and raises early-warning to flights that greatly affect the complexity with the combination of complexity measure raised by Marczyk[18] and OntoSpace software [19].

\section{RESEARCH METHOD}

The metrization analysis system on air traffic complexity based on complexity measure can be achieved by the following steps:

\subsection{Phase Space Establishment}

Suppose simulated data contain $n$ variables and $m$ sample points, all samples matrix of simulated data shows as following:

$$
X=\left[\begin{array}{c}
X_{1} \\
X_{2} \\
\mathrm{M} \\
X m
\end{array}\right]=\left[\begin{array}{cccc}
x_{11} & x_{12} & \mathrm{~L} & x_{1 n} \\
x_{21} & x_{22} & \mathrm{~L} & x_{2 n} \\
\mathrm{M} & \mathrm{M} & \mathrm{M} & \mathrm{M} \\
x_{m 1} & x_{m 2} & \mathrm{~L} & x_{m n}
\end{array}\right]
$$

in which row vector $X_{i}$ stands for the $i$ th simulated data sample.

The study results need to contain $n$ variables as same, whose data are not required to be equal time series with simulated data, nor need they to be sampled at fixed intervals; therefore it saves vacancy-filling when there are different lengths of data sequence or vacancy in middle data. All samples matrix of simulated data shows as following:

$$
Y=\left[\begin{array}{c}
Y_{1} \\
Y_{2} \\
\mathrm{M} \\
Y m
\end{array}\right]=\left[\begin{array}{cccc}
y_{11} & y_{12} & \mathrm{~L} & y_{1 n} \\
y_{21} & y_{22} & \mathrm{~L} & y_{2 n} \\
\mathrm{M} & \mathrm{M} & \mathrm{M} & \mathrm{M} \\
y_{m 1} & y_{m 2} & \mathrm{~L} & y_{m n}
\end{array}\right]
$$

in which row vector $Y_{i}$ stands for the $i$ th simulated data sample.

\subsection{Information Analysis of Phase Space}

\subsubsection{Unary Statistics Analysis}

1) Calculation of Entropy of Information

In the phase space of the established system, the information it contains is calculated quantitatively through the measure of Entropy of information or Shannon Entropy [20]. While the non-linear relativity is calculated quantitatively by generalized correlation coefficient based on mutual information measure.

Suppose the discrete random variable,

$X=\left\{x_{1}, x_{2}, \mathrm{~L}, x_{n}\right\}$

were the given information source, with its appearing rate

$p_{k}=P\left(x_{k}\right),(k=1,2, \mathrm{~L}, n)$ and $\sum_{k=1}^{n} p_{k}=1$,

So the information source $X$ could be expressed as

$H(X)=-\sum_{k=1}^{n} p_{k} \log _{2} p_{k}$

2) K-S Ill-conditioned Calculation

This article deploys K-S test to tell whether the data obey normal distribution and whether the test data meet the requirements. K-S test usually requires working out cumulative distribution functions of the comparable two groups of observation data first, and then to make a judgement on the maximum $\mathrm{D}$ in the absolute values of difference of the cumulative distribution functions.

$$
D=\max _{1 \leq i \leq n}\left\{\left|F\left(X_{i}\right)-F_{0}\left(X_{i}\right)\right|,\left|F\left(X_{i-1}\right)-F_{0}\left(X_{i}\right)\right|\right\}
$$

\subsubsection{Binary Statistics Analysis}

1) Entropy of Mutual Information:

Entropy of information is a concept used in information theory to measure information. Positive correlation is found between the uncertainty of variables and corresponding entropy. The more ordered one system is, the lower its information entropy is and vice versa.

Entropy of mutual information between variable $X$ and $Y$ are defined as: 


$$
H(X, Y)=-\sum_{x} \sum_{y} p(x, y) \log _{2} p(x, y)
$$

\section{2) Generalized Correlation Coefficient}

The non-linear relativity among phase space variables is calculated quantitatively through generalized correlation coefficient based on mutual information measure [21]. Generalized correlation coefficient employs mutual information measure on the analysis of messages implied in two random variables, which enables quantified relativity between the liner and the non-linear superior to traditional Pearson correlation, Spearman correlation coefficients, and Kendall' $\tau$ coefficient. Generalized correlation coefficient is defined as following:

$$
\lambda(X, Y)=\sqrt{1-\exp (-2 * I(X, Y))}
$$

Among which

$$
I(X, Y)=\sum_{A} \sum_{B} p(x, y) \log _{2} \frac{p(x, y)}{p(x) p(y)}
$$

is mutual information measuring the relativity between two random variables statistics. $\lambda$ ranges from 0 to 1 , where $\lambda=1$ stands for strong relativity and $\lambda=0$ stands for no relativity.

\subsection{Overall Information Structure Cognition}

\subsubsection{Fuzzy Disturbance Rules}

According to Zadeh's Principal of Incompatibility [22], the more uncertain a complex system is, the harder to describe it precisely. Therefore, with the help of dividing phase space into fuzzy units in accordance to a certain fuzzy level, fuzzy dependent rules can be quickly and effectively extracted from variables of complex system; while the fuzzy level could be level 3, level 5 or level 7, depending on Data Granularity. After the division of fuzzy units, each sample point belongs to one fuzzy unit, which is every sample point's precise value transforms to one equivalent fuzzy state vector. Phase space with effective information and structure comes out after excluding phase space diagrams of high entropy of information and low relativity.

\subsubsection{System Map}

System Map [23] is used to describe the overall information structure of system on the basis of all the fuzzy rules calculation. System Map is a triple, in which:

\section{Nodes $V$}

$V=\left\{v_{1}, v_{2}, \mathrm{~L}, v_{n}\right\}$ stands for variable set.

\section{Links $L$}

Links $L$ means a map.

$$
L:\left(V_{i}, V_{j}\right) \rightarrow R_{i j}
$$

$R_{i j}$ stands for the union of all fuzzy rules of $V_{i}$ and $V_{j} \cdot V_{i}$, $V_{j} \in V$

\section{Connectors $C O N$}

Connectors $C O N$ means a map

$C O N:\left(V_{i}, V_{j}\right) \rightarrow\left(\lambda_{i j}, e_{i j}\right)$

$\lambda_{i j}$ stands for the generalized correlation coefficient between $V_{i}$ and $V_{j}$. While $e_{i j}$ stands for entropy of phase space composed of $V_{i}$ and $V_{j}$,

$\lambda_{i j}=\sqrt{1-\exp \left(-2 I\left(V_{i}, V_{j}\right)\right)}$

in which $I\left(V_{i}, V_{j}\right)$ is mutual information.

Degree of nodes (variables) in system map stands for their connection degree with other variables, in which the viable with biggest degree is called Hub. There are two ways to define degree:

\section{Connectivity Degree}

The connectivity degree of the $i$ th variable is defined as $D_{i}=\sum_{j} \operatorname{Link}_{j i}$

\section{Correlation Norm,}

The correlation norm of the $i$ th variable is defined as $\lambda_{i}=\sqrt{\sum_{j} \lambda_{j i}^{2}}$.

\subsection{System Evaluation Index}

\subsubsection{Total Entropy of System Information}

Total entropy of system information $T_{E}$ equals to the sum of all variables information entropy divides the mutual information entropy of any two variables.

$$
T_{E}=\sum_{i} H\left(X_{i}\right)-\sum_{i<j}\left(H\left(X_{i}, X_{j}\right)\right)
$$

\subsubsection{The Complexity of System $C$}

The complexity of system $C$ is defined as

$C=\frac{2 T_{E}}{N \xi}, \quad \xi=\sqrt{\frac{\rho_{L}+1}{2}}$

Among which $N$ stands for active nodes, $\xi$ is the correction coefficient which connects intensity in the system map, $\rho_{L}$ means connection intensity in system map which equals to the ratio of detected incidence number and maximum incidence number of the given active node in the system map. 


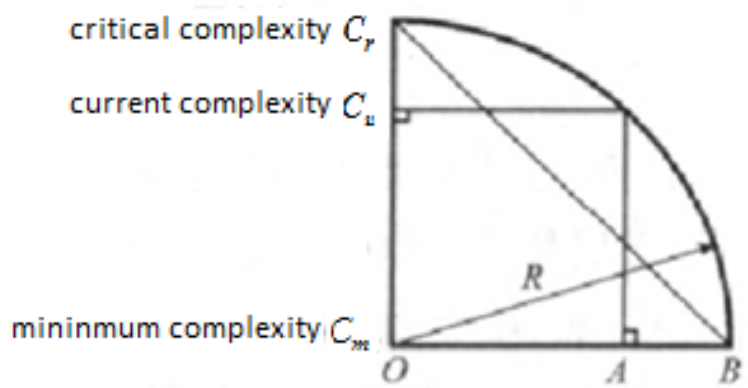

Fig. (1). Calculation method of complexity system robustness.

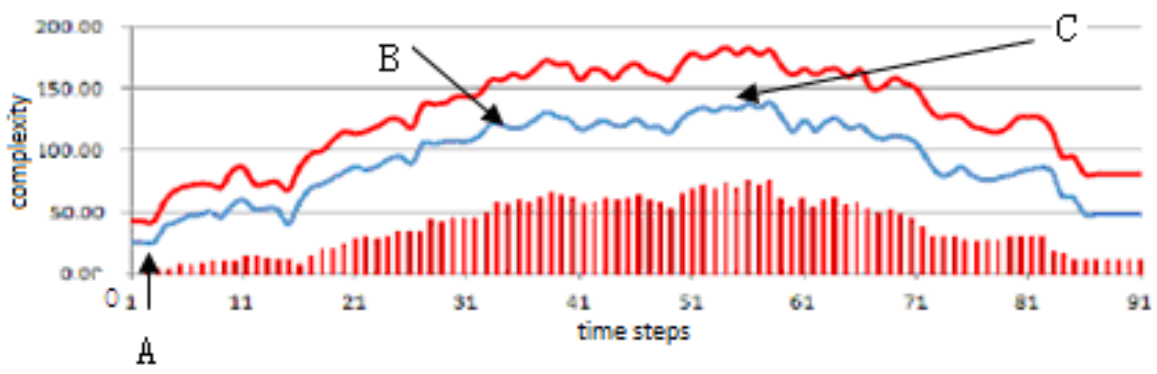

Fig. (2). Evolution process of system complexity.

\subsubsection{Critical Complexity}

Critical complexity means the maximum complexity that maintains structural relation of the system.

\subsubsection{System Robustness:}

Topology structure robustness of system map is an important property of system map, which is a function of critical complexity and lowest complexity according to the present complexity of system map. This article employs circular weighted method to calculate robustness index of system with the idea of penalty function.

As the Fig. (1) shows, $C_{r}$ is the critical complexity of system, $C_{u}$ the present complexity of system and $C_{m}$ the lowest complexity of system. The path is a circular arc with its centre $O$ and its radius $r=d_{O B}=C_{r}-C_{m}$; and $C_{u}$ is made a mapping on the arc and projected to $O B$. The present robustness of system is expressed as $W=\frac{d_{O A}}{d_{O B}}$

\section{CASE ANALYSES}

\subsection{Processing the Acquired Data}

This article selects the radar data at peak operation periods of an airport in Western China, and obtained each flight's longitude, latitude, barometric height, speed and heading angle a total of five parameters after processing the data with analysis software. In this case, there are 14 flights a total of 70 parameters, spanning 5,550 sample points at 5second interval. With 60 sample points as time window, the complexity and entropy of each time window are calculated, among which of key period and key flights are under particular analysis.

\subsection{Dynamic Evolution Process of Traffic Complexity}

According to the calculated results as Fig. (2) shows:

1 The trend of system complexity appears to have been low-high-low; while it hit the peak at Step58 (point C), followed with gradual decline.

2 As Fig. (2) show, from its nadir (point A) to zenith (point C), system complexity and total entropy rockets $448 \%$ and $2136 \%$ respectively, while system robustness plunges $25 \%$.

As Fig. (3) shows, system robustness mostly remains under the threshold of $80 \%$, indicating that system health of the period is below average with big, chaotic traffic flow. Based on the statistics, supervisors are supposed to remind controllers timely to rise to the occasion with fully preparation.

Table 1 tells changes of system structure and system index during the lowest complexity period, high complexity period and highest complexity period. With the advance of complexity, the number of system effective coefficient and rules increase quickly. Effective coefficients increases from 14 to 62 and rules soars from 91 to 1827 , with change rate during highest and lowest complexity periods hitting $342.86 \%$ and $1907.69 \%$ respectively. The great change also 


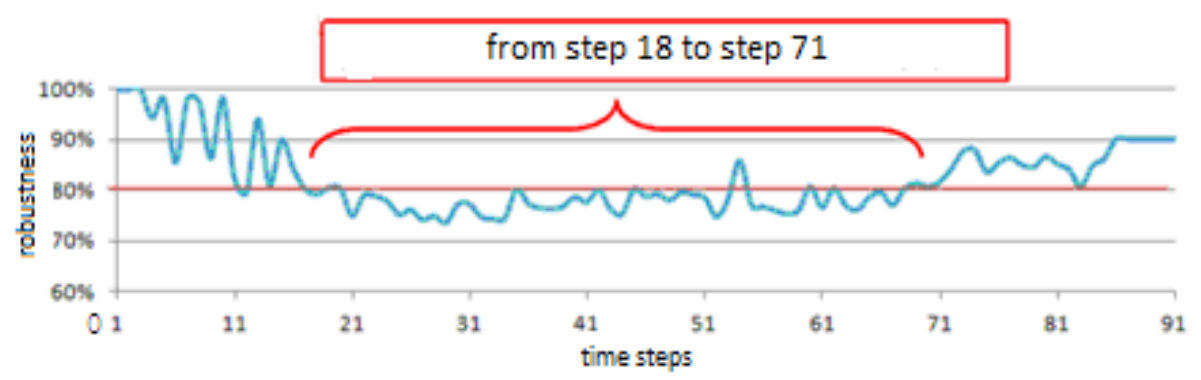

Fig. (3). Evolution process of system robustness.

Table 1. System structure and system indicators at different times.

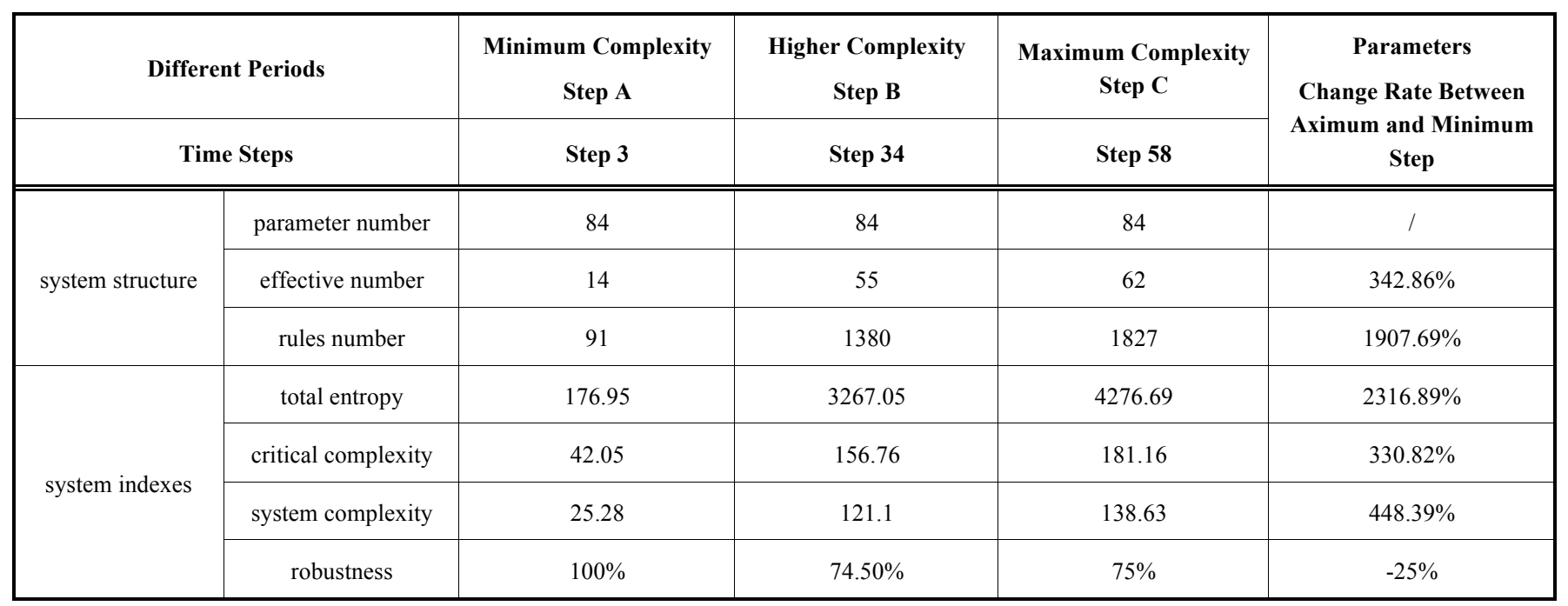

happens to system index, with the change rate of total entropy, critical complexity, and system complexity during highest and lowest complexity period hitting $2316.89 \%$, $330.82 \%$, and $448.39 \%$ respectively. It can be concluded that system complexity shares high uniformity with parameters involved in the system structure.

\subsection{Analysis of Complexity Causes}

It is the change of parameters of flights themselves and positions of aircraft that attribute to the change of traffic flow complexity, for which recognizing key flights that advance traffic complexity is of great necessity. Fig. (4) lists the top 5 key flights that attribute most to complexity over all periods. At the high complex period (Step 34), flight 2097218 ranks No.1 accounting 12\% and flight 7865346 ranks No.4. At highest complex period (Step58), flight 7865346 ranks No.1 by $11 \%$ but flight 2097218 descends to No.3 by $11 \%$. This article offers an analysis method of complexity which enables recognition of key flights that leads to more complex traffic. For such flights controllers shall pay high attention to them and conduct adjustment priority scheduling if necessary in order to lower the whole system's complexity.

\subsection{Crisis Early-Warning Based on Volatility of Com- plexity and Robustness}

By the means of complexity analysis proposed in this article, as Fig. (5) shows, as traffic system appears highly volatility, and so does its robustness, big uncertainty dominates the system at the moment, signalling potential crisis. In Fig. (5), when visible changes showed in complexity, corresponding robustness of system shows troughs and crests. So based on the volatility of traffic system complexity, supervisors could remind controllers to notice aerospace condition at the moment and be prepared if it get worse.

\section{CONCLUSIOON}

After calculation of 14 flights a total of 70 parameters with 5550 sample points as time span, employing analysis of system as a whole instead of subjective hypothesis, this article solves the problem quantified system complexity and health evaluation. Studies show the method this article offers could figure out changes of system complexity from complicated and uncertain data, and after recognizing condition coefficient relative to system robustness with robustness technical index, it can point out the flights which exert heavy influence to the system robustness. Without pre-treatment of 


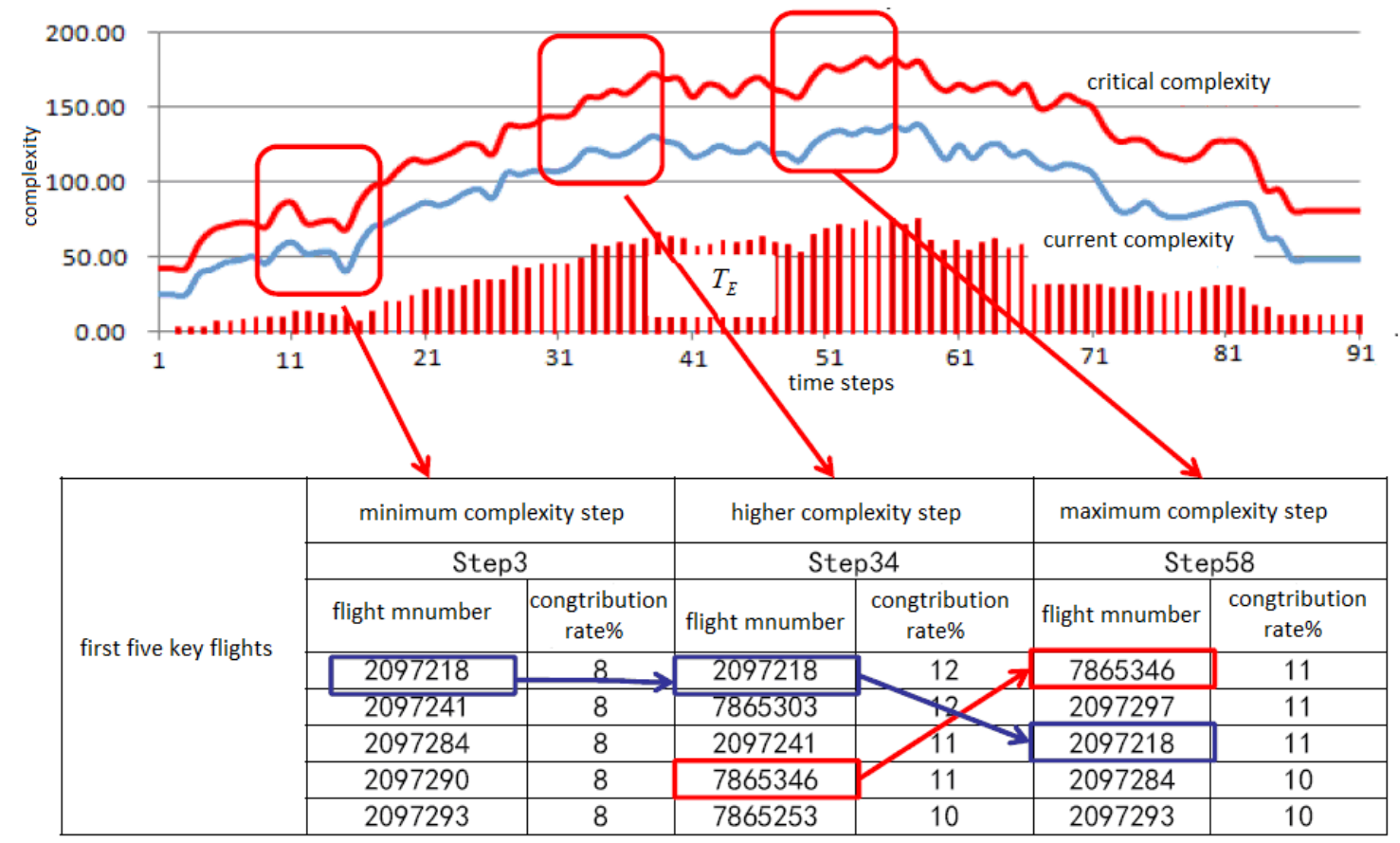

Fig. (4). System complexity contribution figure of different flights in the different periods.

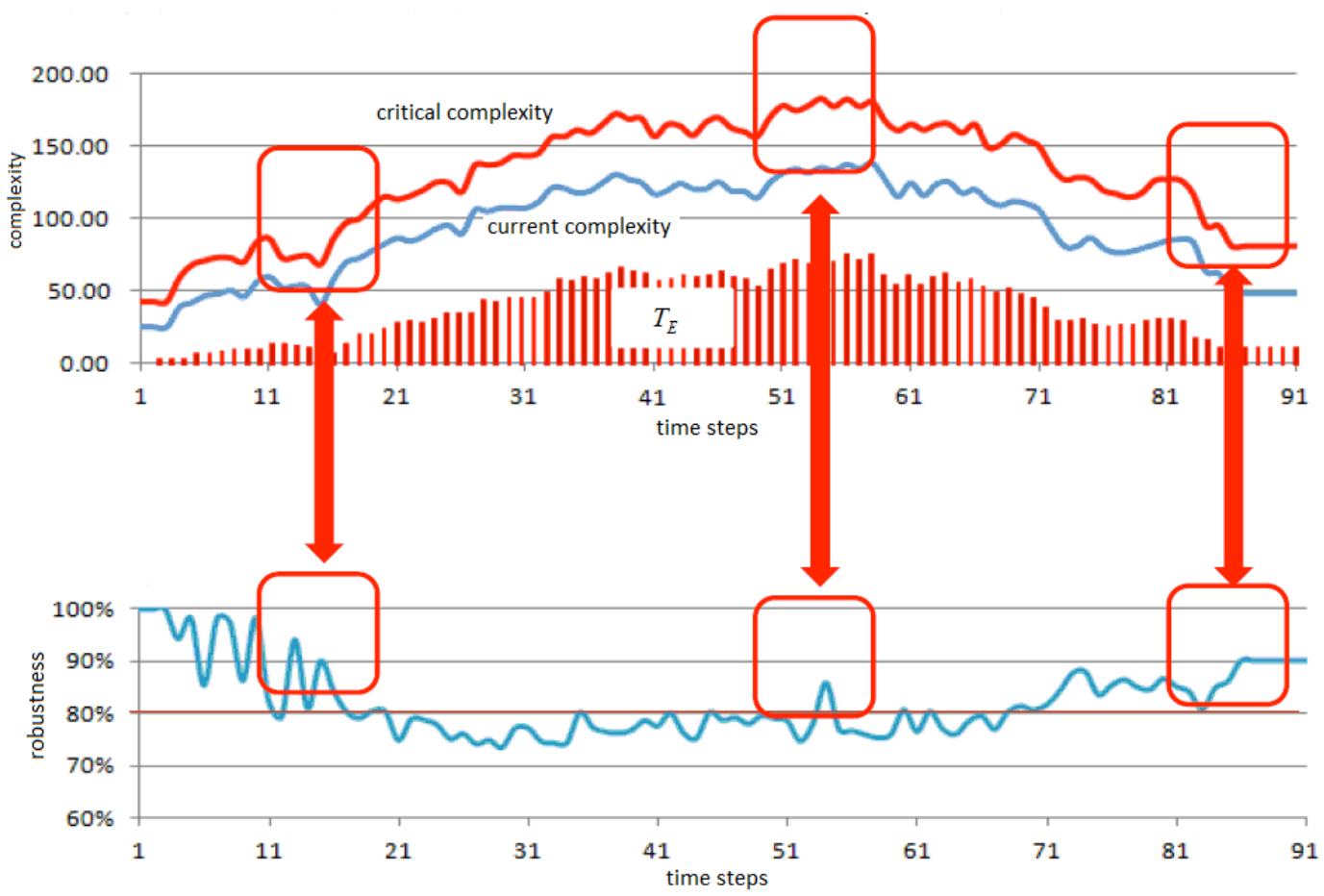

Fig. (5). Relationship graph of traffic flow complexity, robustness and flight early warning.

data or any requirement of samples'distribution, this method could analysis the system in terms of the overall picture saving subjective factors, which could be used to deal with incomplete, small sample data.

\section{CONFLICT OF INTEREST}

The authors confirm that this article content has no conflicts of interest. 


\section{ACKNOWLEDGEMENTS}

This work is supported by the Joint Funds of the National Natural Science Foundation of China and the Civil Aviation Administration of China (Grant No. U1233105, 60832012), partially supported by grant J2010-03, J2014-93 of Science and Technology Fund of CAFUC

\section{REFERENCE}

[1] Laudeman I V, Shelden S G, Branstrom R, et al. 1998 Dynamic density: an air traffic management metric. NASA-TM-1998-11226.

[2] Delahaye D, Puechmorel S. 2000 Air traffic complexity: towards intrinsic metrics. //Proceedings of the 3rd USA/ Europe ATM R\&D Seminar, 1-11.

[3] Wyndemere.1996 An Evaluation of Air Traffic Control Complexity. NASA-CR-202457.

[4] Mogford R H, Guttman J A, Morrow S L, et al. 1995 the Complexity Construct in Air Traffic Control: A review and Synthesis of the Literature. DOT/FAA/CT-TN95/22.

[5] The measure of air traffic control sector complexity for the en route environment: phaseII experiment plan. 2008 FAA.

[6] Delahaye D, Puechmorel S, et al. 2002 A new air traffic complexity metric based on dynamical system modelization. //Proceedings of the 21st Digital Avionics Systems Conference, 1:4A2-1-4A2-12.

[7] Delahaye D, Puechmorel S, Hansan R J, et al. 2004 Air traffic complexity map based on nonlinear dynamical systems. Air Traffic Control Quarterly, 12(4): 367-388.

[8] S.B. Amor, Dac HT, Bui M. Investigating air traffic control dynamics using random cellular automata. // Proceedings of the $5^{\text {th }}$ EUROCONTROL Innovative Research Workshop Exhibition, 2006, 139-143.

[9] K. Lee, E. Feron, A. Pritchett. Describing airspace complexity: airspace response to disturbances. Journal of Guidance, Control and Dynamics, 132(1): 2009, pp. 210-22.

[10] J. Zhang, M.H. Hu, C. Zhang. Complexity research in air traffic management. Acta Aeronautica Et Astronautica Sinica, 30(11): 2009, 2132-42.
[11] Klein A, Rodgers M D, Leiden K. 2009 Simplified dynamic density: a metric for dynamic airspace configuration and nextgen analysis. //IEEE, Proceedings of the $28^{\text {th }}$ Digital Avionics Systems Conference, Piscataway, New Jersey: IEEE, 2.D.3-1-2.D.3-12.

[12] Bui M. 2005 Research activities of the complx systems modeling laboratory. //Proceedings of the 4th EUROCONTROL Innovative Research Workshop Exhibition, 67-72.

[13] Erwan Salaum, Maxime Gariel, Adan E Vela, et al. 2010 Airspace complexity estimations based on data-driven flow modelling. //Proceedings of the Navigation, and Control Conference, 8:2-5.

[14] Zhu Xiao-hui, Zhang Jun. Air traffic complexity evaluation method based on probabilistic trajectory prediction. Systems Engineering and Electronics, 36(2): 2014, pp. 300-5.

[15] Prandini M, Piroddi L, Puechmorel S, et al. 2011 Toward air traffic complexity assessment in new generation air traffic management systems. IEEE Trans. on Intelligent Transportation Systems, 12(3):809-18.

[16] Lempel A, Ziv J. 1976 On the complexity of finite sequences. IEEE Transactions on Information Theory, IT-22(1): 75-81.

[17] J Marczyk, B Deshpande, 2006 Measuring and Tracking Complexity in Science.// International Conference on complex systems Boston, USA.

[18] J Marczyk, 1999 Principles of Simulation Based Computer-Aided Engineering, FIM Publications, Madrid.

[19] Soyotec Inc. Ontospace 2010 User Guide .New York: Soyotec Inc.

[20] Y.E. Fang, L. Yuyang, M. Jacek. Complexity based model credibility validation in research of occupant restraint system simulation. //The fifth China top BBS system modelling and direction is technology, Beijing 2010.

[21] L. Shu-cheng, P. Xin, W. Wei et al. Complexity based robustness analysis for turbulence model in torque converters' flow field simulation. Journal of Jilin University (Engineering and Technology Edition), 42(3): 2013, pp. 613-8.

[22] L.A. Zadeh. 1979 Fuzzy sets and information granularity. Advances in Fuzzy Set Theory and Applications, North-Holland, Amsterdam, 3-18.

[23] C.D. Stylios, P.P. Groumpos. Fuzzy Cognitive Map in modelling Supervisory Control Systems. Journal of Intelligent and Fuzzy Systems, 18(2): 2000, pp. 83-98.

(C) Dong Bing; Licensee Bentham Open.

This is an open access article licensed under the terms of the Creative Commons Attribution Non-Commercial License (http://creativecommons.org/licenses/by-nc/3.0/) which permits unrestricted, non-commercial use, distribution and reproduction in any medium, provided the work is properly cited. 